\title{
Chemical Analyses of Ground Water in the Carson Desert near Stillwater, Churchill County, Nevada, 2005
}

By DeEtta Fosbury', Mark Walker ${ }^{2}$, and Lisa L. Stillings ${ }^{3}$

Open-File Report 2008-1201

U.S. Department of the Interior

U.S. Geological Survey

\footnotetext{
${ }^{1}$ AMEC Earth and Environmental Inc., Portland OR 97224

${ }^{2}$ University of Nevada, Natural Resources and Environmental Sciences Department, Reno, NV 89557

${ }^{3}$ U.S. Geological Survey, MS176, Mackay School of Earth Science and Engineering, University of Nevada, Reno, NV 89557
} 


\section{U.S. Department of the Interior \\ DIRK KEMPTHORNE, Secretary}

\section{U.S. Geological Survey \\ Mark D. Myers, Director}

U.S. Geological Survey, Reston, Virginia 2008

For product and ordering information:

World Wide Web: http://www.usgs.gov/pubprod

Telephone: 1-888-ASK-USGS

For more information on the USGS - the Federal source for science about the Earth,

its natural and living resources, natural hazards, and the environment:

World Wide Web: http://www.usgs.gov

Telephone: 1-888-ASK-USGS

Suggested citation:

Fosbury, D., Walker, M., and Stillings, L., 2008, Chemical analysis of ground water in the Carson Desert near Stillwater, Churchill County, Nevada, 2005: U.S. Geological Survey Open-File Report 2008-1201 [http://pubs.usgs.gov/of/2008/1201/].

Any use of trade, product, or firm names is for descriptive purposes only and does not imply endorsement by the U.S. Government.

Although this report is in the public domain, permission must be secured from the individual copyright owners to reproduce any copyrighted material contained within this report. 


\section{Contents}

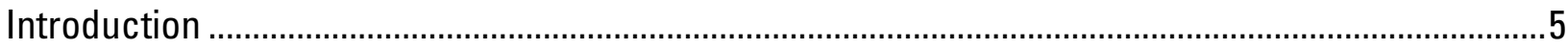

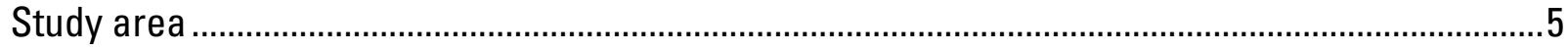

Sampling procedures................................................................................................................

Chemical data for ground water samples...........................................................................................

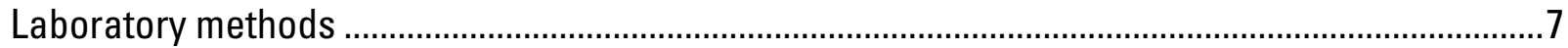

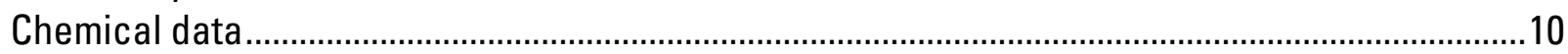

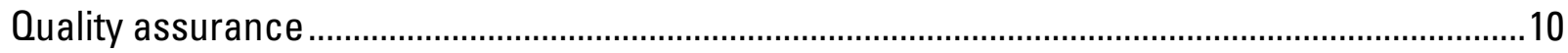

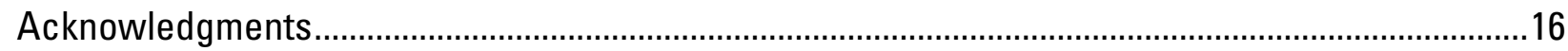

References Cited .......................................................................................................................

\section{Figures}

Figure 1. Location of the study area in Churchill County, NV USA....................................................

\section{Tables}

Table 1: Locations and depths of wells included in the study..........................................................

Table 2: Analytical methods used by Nevada State Health Laboratory .......................................... 9

Table 3: Chemical analyses of well water samples ..........................................................................11

Table 4. Quality assurance data. .................................................................................................. 


\section{Conversion Factors}

\section{SI to Inch/Pound}

\begin{tabular}{lll}
\hline \multicolumn{1}{c}{ Multiply } & \multicolumn{1}{c}{ By } & \multicolumn{1}{c}{ To obtain } \\
\hline Length & & \\
\hline meter $(\mathrm{m})$ & 3.281 & foot $(\mathrm{ft})$ \\
kilometer $(\mathrm{km})$ & 0.6214 & mile $(\mathrm{mi})$ \\
\hline Volume & & \\
\hline liter $(\mathrm{L})$ & 0.2642 & gallon $(\mathrm{gal})$ \\
\hline Mass & & \\
\hline milligram $(\mathrm{mg})$ & $3.527 \times 10^{-5}$ & ounce, avoirdupois $(\mathrm{oz})$
\end{tabular}

Temperature in degrees Celsius $\left({ }^{\circ} \mathrm{C}\right)$ may be converted to degrees Fahrenheit $\left({ }^{\circ} \mathrm{F}\right)$ as follows:

${ }^{\circ} \mathrm{F}=\left(1.8 \times{ }^{\circ} \mathrm{C}\right)+32$

Horizontal coordinate information is in the Universal Transverse Mercator Projection, Zone 11, North American Datum of 1983 (NAD 83)

Altitude, as used in this report, refers to distance above the vertical datum.

Specific conductance is given in microsiemens per centimeter at 25 degrees Celsius $\left(\mu \mathrm{S} / \mathrm{cm}\right.$ at $\left.25^{\circ} \mathrm{C}\right)$.

Color is given in color units (CU); turbidity is given in nephelometric turbidity units (NTU).

Concentrations of chemical constituents in water are given either in milligrams per liter $(\mathrm{mg} / \mathrm{L})$ or micrograms per liter $(\mu \mathrm{g} / \mathrm{L})$. 


\section{Chemical Analyses of Groundwater in the Carson Desert near Stillwater, Churchill County, Nevada}

\section{Introduction}

This report presents the chemical analyses of ground-water samples collected in 2005 from domestic wells located in the Stillwater area of the Carson Desert (fig. 1). These data were evaluated for evidence of mixing with nearby geothermal waters (Fosbury, 2007). That study used several methods to identify mixing zones of ground and geothermal waters using trace elements, chemical equilibria, water temperature, geothermometer estimates, and statistical techniques.

In some regions, geothermal sources influence the chemical quality of ground water used for drinking water supplies. Typical geothermal contaminants include arsenic, mercury, antimony, selenium, thallium, boron, lithium, and fluoride (Webster and Nordstrom, 2003). The Environmental Protection Agency has established primary drinking water standards for these, with the exception of boron and lithium. Concentrations of some trace metals in geothermal water may exceed drinking water standards by several orders of magnitude.

Geothermal influences on water quality are likely to be localized, depending on directions of ground water flow, the relative volumes of geothermal sources and ground water originating from other sources, and depth below the surface from which water is withdrawn. It is important to understand the areal extent of shallow mixing of geothermal water because it may have adverse chemical and aesthetic effects on domestic drinking water. It would be useful to understand the areal extent of these effects.

\section{Study area}

The Stillwater geothermal area is located in the Carson Desert hydrographic area in northcentral Nevada. The community of Stillwater is located about $20 \mathrm{~km}$ east of the city of Fallon, near the center of the Stillwater geothermal area (Garside and Schilling, 1979). Carson Desert is a hydrologically closed basin at the terminus of the Carson River basin (Figure 1).

Four aquifers have been defined in Carson Desert, including a consolidated basalt aquifer and three unconsolidated, basin-fill, sedimentary aquifers. The three basin-fill, sedimentary aquifers are designated as shallow $(0-15 \mathrm{~m}$ or $0-50 \mathrm{ft})$, intermediate $(15-152 \mathrm{~m}$ or $50-500 \mathrm{ft})$, and deep $(>152 \mathrm{~m}$ or $>500 \mathrm{ft})$. The primary basis for these designations is water chemistry, particularly water hardness (Glancy, 1986).

The principal source of recharge to the shallow basin-fill aquifers in the Stillwater area is upwelling water from the intermediate aquifer, and infiltration of surface water from irrigation, which is supplied from the Carson River and Truckee Canal. Three hundred and seventy miles of lined and unlined canals supply water to irrigated fields in the Carson Desert, primarily for alfalfa production (Lico and Seiler, 1994). Previous studies show that residents of the Carson Desert are exposed to high levels of trace elements in the ground water, including arsenic (As) and tungsten (W), which are potentially related to geothermal activity in the area (Lico and Seiler, 1994; Lico et al., 1987; Seiler et al., 2005; Welch and Lico, 1998). These studies have also suggested that high 
concentrations of total dissolved solids (TDS) in Stillwater well-waters are due to evapoconcentration of recharge water.

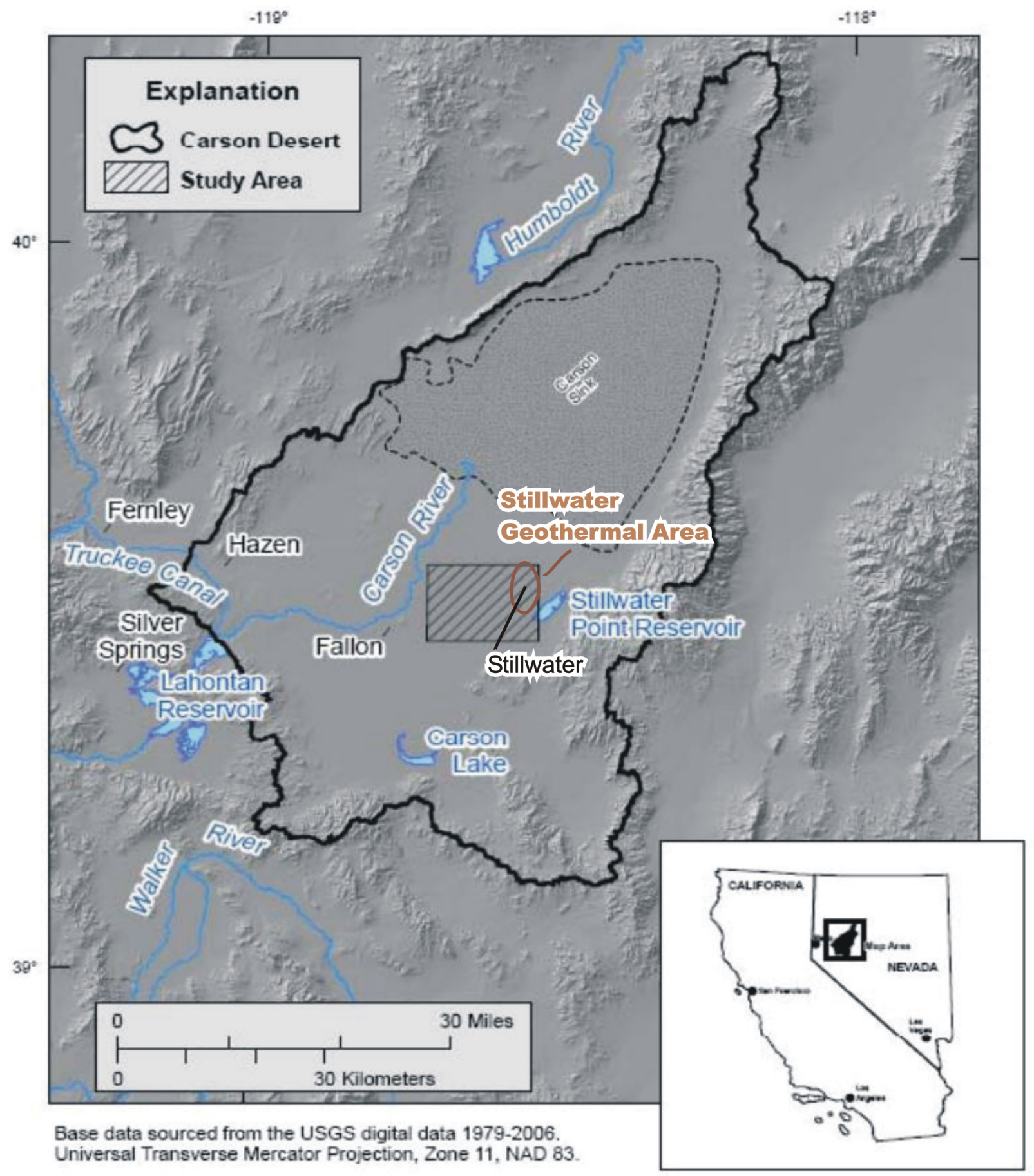

Figure 1. Location of the study area in Churchill County, NV USA. 


\section{Sampling procedures}

Wells in and around Stillwater were sampled once each in June and July of 2005. Table 1 presents location and depth of wells included in the study. Well coordinates were obtained using a geographic positioning system (Garmin GPSmap 76S). Estimated well depths were obtained from interviews with well owners.

For low temperature domestic wells (water temperature $<45^{\circ} \mathrm{C}$ ), samples were taken from the spigot closest to the pump and upstream of any water treatment or storage equipment. A YSI Multiprobe with flow-through cell was used to measure water chemical and physical properties (temperature, $\mathrm{pH}$, specific conductance, and dissolved oxygen) in preparation for sample collection. Each well was purged until readings for temperature, $\mathrm{pH}$, and dissolved oxygen remained constant for 5 minutes. The samples were not filtered. Samples for $\delta^{18} \mathrm{O}$ and $\delta^{2} \mathrm{H}$ analysis were collected in 5-mL glass bottles and sealed with parafilm. Acidified and untreated samples were collected in plastic bottles provided by the Nevada State Health Laboratory. Table 2 lists methods and associated reporting limits.

The YSI Multiprobe and flow-through cell could not be used with hot waters $\left(>45^{\circ} \mathrm{C}\right)$. Hot domestic wells were sampled from the spigot after purging 56-75 liters and temperature remained constant for 5 minutes. Temperatures were measured with a hand-held thermometer. Two samples from a geothermal power plant (P1 and P2) were taken from valves closest to wellheads.

Temperatures were measured by facility personnel. The open-air method of sampling at the power plant resulted in a great deal of steam loss from these samples, which must be considered when interpreting results.

\section{Chemical data for ground water samples}

\section{Laboratory methods}

All sample analyses, excluding isotopes, were performed by the Nevada State Health Laboratory (NSHL), on the campus of the University of Nevada, Reno, in Reno, Nevada. The methods used for each analyte are listed in Table 2. Isotope samples $\left(\delta_{2}^{18} \mathrm{O}\right.$ and $\left.\delta^{2} \mathrm{H}\right)$ were analyzed at the University of Nevada, Reno Stable Isotope Laboratory. Water- $\delta$ H analyses were performed using the method of Morrison et al. (2001). Water- $\delta$ O analyses were performed using the $\mathrm{CO}_{2}$ $\mathrm{H}_{2} \mathrm{O}$ equilibration method of Epstein and Mayeda (1953). One field blank and 4 field duplicates were collected and analyzed for the full suite of analytes, in order to assess for quality assurance. 
Table 1: Locations and depths of wells included in the study

\begin{tabular}{|c|c|c|c|c|c|}
\hline $\begin{array}{c}\text { Well } \\
\text { Designation }\end{array}$ & $\begin{array}{l}\text { UTM Zone } 11 \\
\text { Easting }\end{array}$ & $\begin{array}{l}\text { UTM Zone } 11 \\
\text { Northing }\end{array}$ & Longitude & Latitude & $\begin{array}{c}\text { Total Well } \\
\text { Depth } \mathrm{m}(\mathrm{ft})\end{array}$ \\
\hline 1 & 367195 & 4375744 & -118.545052 & 39.521083 & $9(30)$ \\
\hline 2 & 366536 & 4375907 & -118.552749 & 39.522449 & $10(33)$ \\
\hline 3 & 364752 & 4377825 & -118.573887 & 39.539446 & $15(50)$ \\
\hline 4 & 367102 & 4375991 & -118.546183 & 39.523294 & $12(40)$ \\
\hline 5 & 366821 & 4375846 & -118.549422 & 39.521944 & $15(50)$ \\
\hline 6 & 367087 & 4375912 & -118.546342 & 39.522580 & $11(35)$ \\
\hline 7 & 367253 & 4375857 & -118.544400 & 39.522110 & $11(37)$ \\
\hline 8 & 365029 & 4375524 & -118.570198 & 39.518764 & $8(25)$ \\
\hline 9 & 362985 & 4374752 & -118.593809 & 39.511487 & $9(28)$ \\
\hline 10 & 365762 & 4375064 & -118.561581 & 39.514736 & $8(25)$ \\
\hline 11 & 364688 & 4374498 & -118.573955 & 39.509469 & $310(1017)$ \\
\hline 12 & 364652 & 4375068 & -118.574489 & 39.514598 & $9(31)$ \\
\hline 13 & 366013 & 4376813 & -118.559014 & 39.530529 & $6(20)$ \\
\hline 14 & 365981 & 4376791 & -118.559382 & 39.530325 & $152(500)$ \\
\hline 15 & 363809 & 4374817 & -118.584241 & 39.512204 & $6(20)$ \\
\hline 16 & 363791 & 4374776 & -118.584442 & 39.511832 & $10(34)$ \\
\hline 17 & 364316 & 4378813 & -118.579161 & 39.548276 & $49(160)$ \\
\hline 19 & 357390 & 4372648 & -118.658417 & 39.491627 & $7(22)$ \\
\hline 21 & 355647 & 4370785 & -118.678275 & 39.474556 & $11(36)$ \\
\hline 22 & 358468 & 4372374 & -118.645827 & 39.489337 & $6(19)$ \\
\hline 23 & 361363 & 4371528 & -118.611999 & 39.482188 & $8(25)$ \\
\hline 25 & 355215 & 4370813 & -118.683301 & 39.474736 & $44(145)$ \\
\hline 26 & 356533 & 4372041 & -118.668248 & 39.486017 & $6(20)$ \\
\hline 27 & 356379 & 4372460 & -118.670129 & 39.489765 & $6(20)$ \\
\hline 28 & 356562 & 4372510 & -118.668012 & 39.490246 & $6(20)$ \\
\hline 29 & 356380 & 4372546 & -118.670135 & 39.490540 & $6(20)$ \\
\hline 30 & 356440 & 4371273 & -118.669861 & 39.479074 & $8(25)$ \\
\hline 32 & 355492 & 4372242 & -118.680392 & 39.487653 & $6(20)$ \\
\hline 33 & 355476 & 4370512 & -118.680203 & 39.472069 & $5(15)$ \\
\hline 34 & 358262 & 4372356 & -118.648218 & 39.489141 & $4(14)$ \\
\hline 35 & 356267 & 4370770 & -118.671066 & 39.474525 & $4(13)$ \\
\hline 36 & 357291 & 4373970 & -118.659851 & 39.503517 & $8(25)$ \\
\hline 37 & 357963 & 4374026 & -118.652050 & 39.504133 & $8(25)$ \\
\hline 38 & 366678 & 4377863 & -118.551490 & 39.540090 & $125(410)$ \\
\hline 39 & 366725 & 4378334 & -118.551037 & 39.544339 & $70(230)$ \\
\hline 40 & 366787 & 4378341 & -118.550317 & 39.544412 & $6(20)$ \\
\hline 41 & 366682 & 4377256 & -118.551321 & 39.534623 & $70(230)$ \\
\hline 42 & 363922 & 4372847 & -118.582524 & 39.494478 & $6(20)$ \\
\hline $\mathrm{P} 1$ & 366122 & 4378638 & -118.558114 & 39.546984 & $814(2672)$ \\
\hline $\mathrm{P} 2$ & 366477 & 4378327 & -118.553921 & 39.544238 & $381(1250)$ \\
\hline
\end{tabular}


Table 2: Analytical methods used by Nevada State Health Laboratory

\begin{tabular}{|c|c|c|c|}
\hline Chemical Constituent & Preservative & $\begin{array}{l}\text { Analytical } \\
\text { Method }\end{array}$ & $\begin{array}{c}\text { Laboratory Reporting } \\
\text { Limit }\end{array}$ \\
\hline Alkalinity as $\mathrm{CaCO}_{3}$ & none & $\mathrm{SM}^{1} 2320 \mathrm{~B}$ & $20 \mathrm{mg} / 1$ \\
\hline Antimony & $15 \% \mathrm{HNO}_{3}(5 \mathrm{ml})$ & $\mathrm{EPA}^{2} 200.8$ & $1 \mu \mathrm{g} / 1$ \\
\hline Arsenic & $15 \% \mathrm{HNO}_{3}(5 \mathrm{ml})$ & EPA 200.8 & $3 \mu \mathrm{g} / 1$ \\
\hline Barium & $15 \% \mathrm{HNO}_{3}(5 \mathrm{ml})$ & EPA 200.7 & $0.02 \mathrm{mg} / \mathrm{l}$ \\
\hline Bicarbonate & none & SM $2320 \mathrm{~B}$ & $25 \mathrm{mg} / \mathrm{l}$ \\
\hline Boron & $15 \% \mathrm{HNO}_{3}(5 \mathrm{ml})$ & EPA 200.7 & $0.5 \mathrm{mg} / 1$ \\
\hline Calcium & $15 \% \mathrm{HNO}_{3}(5 \mathrm{ml})$ & EPA 200.7 & $5 \mathrm{mg} / \mathrm{l}$ \\
\hline Carbonate & none & SM $2320 \mathrm{~B}$ & $12 \mathrm{mg} / \mathrm{l}$ \\
\hline Cesium & $15 \% \mathrm{HNO}_{3}(5 \mathrm{ml})$ & EPA 200.8 & $1 \mu \mathrm{g} / 1$ \\
\hline Chloride & none & EPA 300.0 & $250 \mathrm{mg} / \mathrm{l}$ \\
\hline Color & none & SM $2120 \mathrm{~B}$ & $3 \mathrm{CU}$ \\
\hline Copper & $15 \% \mathrm{HNO}_{3}(5 \mathrm{ml})$ & EPA 200.7 & $0.02 \mathrm{mg} / \mathrm{l}$ \\
\hline Electrical Conductivity & none & SM 2510 & $10 \mu \mathrm{S} / \mathrm{cm}$ \\
\hline Fluoride & none & SM 4500 F C & $0.2 \mathrm{mg} / 1$ \\
\hline Hardness & $15 \% \mathrm{HNO}_{3}(5 \mathrm{ml})$ & EPA 200.7 & $33 \mathrm{mg} / \mathrm{l}$ \\
\hline Hydroxide & none & SM $2320 \mathrm{~B}$ & $7 \mathrm{mg} / \mathrm{l}$ \\
\hline Iron & $15 \% \mathrm{HNO}_{3}(5 \mathrm{ml})$ & EPA 200.7 & $0.05 \mathrm{mg} / \mathrm{l}$ \\
\hline Lithium & $15 \% \mathrm{HNO}_{3}(5 \mathrm{ml})$ & SM $3500 \mathrm{Li} \mathrm{B}$ & $0.05 \mathrm{mg} / \mathrm{l}$ \\
\hline Magnesium & $15 \% \mathrm{HNO}_{3}(5 \mathrm{ml})$ & EPA 200.7 & $5 \mathrm{mg} / 1$ \\
\hline Manganese & $15 \% \mathrm{HNO}_{3}(5 \mathrm{ml})$ & EPA 200.7 & $0.02 \mathrm{mg} / \mathrm{l}$ \\
\hline Mercury & $15 \% \mathrm{HNO}_{3}(5 \mathrm{ml})$ & EPA 245.2 & $0.2 \mu \mathrm{g} / 1$ \\
\hline Nitrate + Nitrite as $\mathrm{N}$ & $10 \% \mathrm{H}_{2} \mathrm{SO}_{4}(1 \mathrm{ml})$ & EPA 300.0 & $0.5 \mathrm{mg} / 1$ \\
\hline $\mathrm{pH}$ & none & EPA 150.0 & $\mathrm{n} / \mathrm{a}$ \\
\hline Temperature & none & EPA 150.0 & $\mathrm{n} / \mathrm{a}$ \\
\hline Potassium & $15 \% \mathrm{HNO}_{3}(5 \mathrm{ml})$ & EPA 200.7 & $5 \mathrm{mg} / \mathrm{l}$ \\
\hline Selenium & $15 \% \mathrm{HNO}_{3}(5 \mathrm{ml})$ & EPA 200.8 & $1 \mu \mathrm{g} / 1$ \\
\hline Silica $\left(\right.$ as $\left.\mathrm{SiO}_{2}\right)$ & $15 \% \mathrm{HNO}_{3}(5 \mathrm{ml})$ & EPA 200.7 & $1 \mathrm{mg} / \mathrm{l}$ \\
\hline Sodium & $15 \% \mathrm{HNO}_{3}(5 \mathrm{ml})$ & EPA 200.7 & $25 \mathrm{mg} / \mathrm{l}$ \\
\hline Sulfate & none & EPA 300.0 & $25 \mathrm{mg} / \mathrm{l}$ \\
\hline Thallium & $15 \% \mathrm{HNO}_{3}(5 \mathrm{ml})$ & EPA 200.8 & $0.5 \mu \mathrm{g} / 1$ \\
\hline Total Dissolved Solids & none & SM $2540 \mathrm{C}$ & $25 \mathrm{mg} / \mathrm{l}$ \\
\hline Tungsten & $15 \% \mathrm{HNO}_{3}(5 \mathrm{ml})$ & EPA 200.8 & $1 \mu \mathrm{g} / 1$ \\
\hline Turbidity & none & EPA 180.1 & $0.4 \mathrm{NTU}$ \\
\hline Zinc & $15 \% \mathrm{HNO}_{3}(5 \mathrm{ml})$ & EPA 200.7 & $0.05 \mathrm{mg} / \mathrm{l}$ \\
\hline
\end{tabular}

${ }^{1}$ (American Public Health Association., 1998)

${ }^{2}$ (U.S. Environmental Protection Agency) 


\section{Chemical data}

Chemical data for groundwater samples are presented in Table 3. Samples numbers 1-39 were collected from domestic water wells, while samples P1 and P2 were collected from the Stillwater geothermal power plant.

\section{Quality assurance}

Quality assurance data are presented in Table 4. Analysis of the field blank was below reporting levels, for all analytes. Precision of the duplicate samples was expressed as the percent relative standard deviation (\%RSD), also called the coefficient of variation (eqn. 1; Skoog et al., 2000). Duplicate samples generally showed a \%RSD of $0-5 \%$.

$$
\% R S D=\frac{s}{x} \times 100 \%
$$

Where

$s=$ standard deviation, and

$-$

$x=$ the mean of duplicate samples. 
Table 3. Chemical analyses of ground water samples. Well locations and depth are in Table 1. " $<$ RL" = less than the reporting limit.

\begin{tabular}{|c|c|c|c|c|c|c|c|c|c|c|c|}
\hline Sample & 1 & 2 & 3 & 4 & 5 & 6 & 7 & 8 & 9 & 10 & 11 \\
\hline Field Water Temperature $\left({ }^{\circ} \mathrm{C}\right)$ & 40.1 & 19.8 & 19.7 & 19.0 & 22.0 & 33.2 & 25.9 & 13.8 & 18.0 & 19.12 & 39.7 \\
\hline $\mathrm{pH}$ & 7.78 & 8.21 & 8.19 & 8.22 & 8.23 & 8.02 & 8.19 & 7.98 & 8.12 & 8.27 & 8.06 \\
\hline $\mathrm{pH}$ Temperature $\left({ }^{\circ} \mathrm{C}\right)$ & 17.6 & 18.3 & 17.9 & 18.2 & 19 & 18.4 & 18.1 & 18 & 18.3 & 18.9 & 18.1 \\
\hline Electrical Conductivity $(\mu \mathrm{S} / \mathrm{cm})$ & 8300 & 2000 & 9300 & 1300 & 5100 & 3000 & 2900 & 820 & 6900 & 1900 & 6900 \\
\hline Total Dissolved Solids (mg/L) & 5405 & 1360 & 5328 & 881 & 3028 & 1832 & 1720 & 529 & 4236 & 1145 & 4015 \\
\hline Hardness (mg/L) & 867 & 281 & 165 & 149 & 122 & 514 & 233 & 203 & 663 & 162 & 155 \\
\hline Turbidity (NTU) & 0.2 & 0.2 & 2.7 & 0.2 & 0.2 & 2.6 & 0.2 & 0.2 & 1.3 & 0.2 & 0.2 \\
\hline Color (CU) & 5 & 5 & 50 & 5 & 5 & 7 & 5 & 7 & 7 & 5 & 5 \\
\hline Alkalinity as $\mathrm{CaCO}_{3}(\mathrm{mg} / \mathrm{L})$ & 274 & 297 & 792 & 319 & 246 & 352 & 306 & 189 & 330 & 304 & 85 \\
\hline Antimony ( $\mu \mathrm{g} / \mathrm{L})$ & 1 & 3 & $<\mathrm{RL}$ & 2 & $<R L$ & 2 & 3 & $<R L$ & 4 & 2 & $<\mathrm{RL}$ \\
\hline Arsenic $(\mu \mathrm{g} / \mathrm{L})$ & 94 & 200 & 43 & 190 & 7 & 77 & 160 & 17 & 230 & 310 & $<\mathrm{RL}$ \\
\hline Arsenic III ( $\mu \mathrm{g} / \mathrm{L})$ & 60 & 9 & 12 & $<\mathrm{RL}$ & $<\mathrm{RL}$ & $<\mathrm{RL}$ & $<R L$ & $<\mathrm{RL}$ & 110 & $<\mathrm{RL}$ & $<R L$ \\
\hline Barium (mg/L) & 0.05 & 0.04 & 0.36 & 0.08 & 0.07 & 0.28 & 0.13 & 0.06 & 0.03 & 0.04 & 0.11 \\
\hline Bicarbonate (mg/L) & 334 & 362 & 966 & 389 & 300 & 429 & 373 & 230 & 402 & 371 & 103 \\
\hline Boron (mg/L) & 16 & 2.2 & 18 & 1.7 & 12 & 6.5 & 4.3 & 0.6 & 5.2 & 1.5 & 15 \\
\hline Calcium (mg/L) & 240 & 53 & 25 & 40 & 49 & 140 & 57 & 55 & 84 & 32 & 62 \\
\hline Carbonate (mg/L) & $<\mathrm{RL}$ & $<\mathrm{RL}$ & $<\mathrm{RL}$ & $<\mathrm{RL}$ & $<\mathrm{RL}$ & $<\mathrm{RL}$ & $<\mathrm{RL}$ & $<\mathrm{RL}$ & $<\mathrm{RL}$ & $<\mathrm{RL}$ & $<\mathrm{RL}$ \\
\hline Cesium $(\mu \mathrm{g} / \mathrm{L})$ & $<R L$ & $<R L$ & $<R L$ & $<R L$ & $<R L$ & $<R L$ & $<R L$ & $<R L$ & $<R L$ & $<R L$ & 80 \\
\hline Chloride (mg/L) & 2300 & 260 & 3000 & 170 & 1400 & 710 & 700 & 72 & 1800 & 320 & 2200 \\
\hline Fluoride (mg/L) & 1.3 & 1.1 & 0.83 & 1.4 & 4.5 & 0.6 & 1.2 & 0.4 & 2.3 & 3.1 & 4.1 \\
\hline Hydroxide (mg/L) & $<R L$ & $<R L$ & $<R L$ & $<\mathrm{RL}$ & $<\mathrm{RL}$ & $<R L$ & $<R L$ & $<R L$ & $<R L$ & $<R L$ & $<R L$ \\
\hline Iron (mg/L) & $<\mathrm{RL}$ & $<R L$ & 1.2 & $<\mathrm{RL}$ & 0.44 & 1.3 & 0.08 & 0.09 & 0.36 & $<R L$ & 0.13 \\
\hline Lithium $(\mu \mathrm{g} / \mathrm{L})$ & 1.9 & 0.52 & 1 & 0.31 & 1.7 & 0.68 & 0.61 & 0.1 & 0.65 & 0.43 & 2.1 \\
\hline Magnesium (mg/L) & 65 & 36 & 25 & 12 & $<\mathrm{RL}$ & 40 & 22 & 16 & 110 & 20 & $<R L$ \\
\hline Manganese (mg/L) & 1.3 & 0.09 & 0.13 & 0.07 & 0.05 & 0.66 & 0.05 & 0.16 & 0.14 & $<R L$ & 0.06 \\
\hline Mercury $(\mu \mathrm{g} / \mathrm{L})$ & $<\mathrm{RL}$ & $<\mathrm{RL}$ & $<\mathrm{RL}$ & $<\mathrm{RL}$ & $<\mathrm{RL}$ & $<\mathrm{RL}$ & $<\mathrm{RL}$ & $<\mathrm{RL}$ & $<\mathrm{RL}$ & $<\mathrm{RL}$ & $<\mathrm{RL}$ \\
\hline Nitrate + Nitrite as N (mg/L) & $<\mathrm{RL}$ & $<\mathrm{RL}$ & 1.3 & $<\mathrm{RL}$ & $<\mathrm{RL}$ & $<\mathrm{RL}$ & $<\mathrm{RL}$ & $<\mathrm{RL}$ & $<\mathrm{RL}$ & $<\mathrm{RL}$ & $<\mathrm{RL}$ \\
\hline Potassium (mg/L) & 43 & 20 & 52 & 7 & 22 & 19 & 16 & 7 & 54 & 16 & 21 \\
\hline Selenium ( $\mu \mathrm{g} / \mathrm{L})$ & 8 & 2 & 3 & 1 & 4 & 2 & 3 & $<R L$ & 6 & 2 & 6 \\
\hline Silica (mg/L) & 111 & 60 & 56 & 64 & 94 & 79 & 68 & 34 & 60 & 60 & 75 \\
\hline Sodium (mg/L) & 1500 & 340 & 2000 & 240 & 1100 & 450 & 540 & 99 & 1200 & 360 & 1400 \\
\hline Sulfate $(\mathrm{mg} / \mathrm{L})$ & 800 & 400 & $<R L$ & 98 & 210 & 170 & 150 & 130 & 680 & 180 & 160 \\
\hline Thallium ( $\mu \mathrm{g} / \mathrm{L})$ & $<\mathrm{RL}$ & $<\mathrm{RL}$ & $<\mathrm{RL}$ & $<\mathrm{RL}$ & $<\mathrm{RL}$ & $<R L$ & $<R L$ & $<\mathrm{RL}$ & $<\mathrm{RL}$ & $<\mathrm{RL}$ & $<\mathrm{RL}$ \\
\hline Tungsten $(\mu \mathrm{g} / \mathrm{L})$ & 12 & 5 & 32 & 9 & 58 & 5 & 5 & 2 & 4 & 3 & 44 \\
\hline Zinc $(\mathrm{mg} / \mathrm{L})$ & $<R L$ & $<R L$ & $<R L$ & $<R L$ & $<R L$ & $<R L$ & $<R L$ & $<R L$ & $<\mathrm{RL}$ & $<R L$ & $<R L$ \\
\hline$\delta^{18} \mathrm{O}$ (VSMOW \%о) & -11.53 & -10.61 & -11.69 & -10.79 & -11.91 & -11.03 & -10.84 & -8.68 & -10.49 & -10.15 & -12.51 \\
\hline$\delta^{2} \mathrm{H}($ VSMOW \%o) & -102 & -89.5 & -104.8 & -90.8 & -103.7 & -95.7 & -93.8 & -81 & -91.1 & -86.2 & -109.7 \\
\hline
\end{tabular}


Table 3. Chemical analyses of well water samples (continued).

\begin{tabular}{|c|c|c|c|c|c|c|c|c|c|c|c|}
\hline Sample & 12 & 13 & 14 & 15 & 16 & 17 & 19 & 21 & 22 & 23 & 25 \\
\hline Field Temp $\left({ }^{\circ} \mathrm{C}\right)$ & 17.0 & 19.4 & 65.0 & 16.0 & 16.1 & 21.612 & 13.8 & 22.5 & 18.2 & 18.2 & 20.1 \\
\hline $\mathrm{pH}(\mathrm{SU})$ & 8.29 & 8.02 & 7.8 & 8.08 & 7.93 & 8.01 & 8.16 & 7.84 & 8.27 & 7.95 & 8.51 \\
\hline pH Temperature $\left({ }^{\circ} \mathrm{C}\right)$ & 18.5 & 17.6 & 18.3 & 16.9 & 17.6 & 18.9 & 18.7 & 17.8 & 17.7 & 16.8 & 20.7 \\
\hline Electrical Conductivity ( $\mu \mathrm{S} / \mathrm{cm})$ & 630 & 5000 & 7000 & 820 & 980 & 7000 & 1100 & 1700 & 1300 & 3400 & 3600 \\
\hline Total Dissolved Solids (mg/L) & 392 & 3332 & 4108 & 572 & 660 & 3988 & 736 & 1204 & 856 & 2088 & 2170 \\
\hline Hardness (mg/L) & 180 & 526 & 207 & 231 & 254 & 174 & 232 & 573 & 195 & 651 & 16.5 \\
\hline Turbidity (NTU) & 0.2 & 0.2 & 0.6 & 0.2 & 0.2 & 0.2 & 0.2 & 0.2 & 0.2 & 0.45 & 0.2 \\
\hline Color (CU) & 5 & 7 & 5 & 20 & 7 & 5 & 5 & 20 & 7 & 7 & 80 \\
\hline Alkalinity as $\mathrm{CaCO}_{3}(\mathrm{mg} / \mathrm{L})$ & 176 & 496 & 100 & 219 & 198 & 153 & 255 & 361 & 221 & 526 & 749 \\
\hline Antimony $(\mu \mathrm{g} / \mathrm{L})$ & 2 & 3 & $<R L$ & $<R L$ & $<R L$ & $<R L$ & $<R L$ & $<R L$ & $<\mathrm{RL}$ & $<R L$ & $<\mathrm{RL}$ \\
\hline Arsenic ( $\mu \mathrm{g} / \mathrm{L})$ & 40 & 370 & $<\mathrm{RL}$ & 26 & 19 & 9 & 15 & 17 & 24 & 170 & $<\mathrm{RL}$ \\
\hline Arsenic III ( $\mu \mathrm{g} / \mathrm{L})$ & $<R L$ & 230 & $<R L$ & $<R L$ & $<R L$ & 11 & $<R L$ & $<R L$ & $<R L$ & $<R L$ & $<R L$ \\
\hline Barium (mg/L) & 0.08 & 0.03 & 0.16 & 0.14 & 0.12 & 0.15 & 0.11 & 0.15 & 0.09 & 0.13 & 0.04 \\
\hline Bicarbonate (mg/L) & 213 & 605 & 122 & 267 & 241 & 186 & 311 & 440 & 269 & 642 & 842 \\
\hline Boron (mg/L) & 0.5 & 8.6 & 16 & 0.7 & 0.7 & 14 & 0.9 & 1.1 & 1.5 & 1.6 & 11 \\
\hline Calcium (mg/L) & 49 & 100 & 83 & 66 & 72 & 58 & 70 & 170 & 55 & 160 & $<\mathrm{RL}$ \\
\hline Carbonate (mg/L) & $<R L$ & $<\mathrm{RL}$ & $<\mathrm{RL}$ & $<\mathrm{RL}$ & $<R L$ & $<\mathrm{RL}$ & $<\mathrm{RL}$ & $<\mathrm{RL}$ & $<\mathrm{RL}$ & $<\mathrm{RL}$ & 36 \\
\hline Cesium ( $\mu \mathrm{g} / \mathrm{L})$ & $<R L$ & $<R L$ & 160 & $<R L$ & $<R L$ & $<R L$ & $<R L$ & $<R L$ & $<\mathrm{RL}$ & $<R L$ & $<\mathrm{RL}$ \\
\hline Chloride (mg/L) & 52 & 990 & 2200 & 45 & 86 & 2300 & 100 & 190 & 180 & 840 & 750 \\
\hline Copper (mg/L) & $<R L$ & $<R L$ & $<R L$ & $<R L$ & $<R L$ & $<\mathrm{RL}$ & $<R L$ & $<R L$ & $<\mathrm{RL}$ & $<R L$ & $<\mathrm{RL}$ \\
\hline Fluoride (mg/L) & 0.7 & 2.2 & 4.5 & 0.6 & 0.3 & 2.4 & 0.4 & 0.4 & 0.3 & 0.8 & 2.1 \\
\hline Hydroxide (mg/L) & $<R L$ & $<R L$ & $<R L$ & $<R L$ & $<R L$ & $<R L$ & $<R L$ & $<R L$ & $<R L$ & $<R L$ & $<R L$ \\
\hline Iron (mg/L) & $<\mathrm{RL}$ & $<\mathrm{RL}$ & 0.08 & $<\mathrm{RL}$ & $<\mathrm{RL}$ & 0.15 & $<\mathrm{RL}$ & $<\mathrm{RL}$ & 0.05 & 0.05 & $<\mathrm{RL}$ \\
\hline Lithium $(\mu \mathrm{g} / \mathrm{L})$ & 0.1 & 1 & 1.8 & 0.08 & 0.08 & 1.6 & 0.07 & 0.14 & 0.08 & 0.21 & 0.07 \\
\hline Magnesium (mg/L) & 14 & 67 & $<\mathrm{RL}$ & 16 & 18 & 7 & 14 & 36 & 14 & 61 & $<\mathrm{RL}$ \\
\hline Manganese (mg/L) & 0.1 & 0.09 & 0.07 & $<\mathrm{RL}$ & 0.05 & 0.26 & 0.37 & 0.16 & 0.21 & $<\mathrm{RL}$ & $<\mathrm{RL}$ \\
\hline Mercury ( $\mu \mathrm{g} / \mathrm{L})$ & $<R L$ & $<R L$ & $<R L$ & $<R L$ & $<R L$ & $<\mathrm{RL}$ & $<R L$ & $<R L$ & $<R L$ & $<R L$ & $<\mathrm{RL}$ \\
\hline Nitrate + Nitrite as N (mg/L) & 0.6 & 6 & $<\mathrm{RL}$ & 8.9 & 9.9 & $<\mathrm{RL}$ & 1.3 & 16 & 0.9 & 0.6 & $<\mathrm{RL}$ \\
\hline Potassium (mg/L) & 8 & 31 & 31 & 10 & 8 & 22 & 8 & $<\mathrm{RL}$ & 6 & 12 & 13 \\
\hline Selenium ( $\mu \mathrm{g} / \mathrm{L})$ & $<R L$ & 3 & 3 & $<R L$ & 2 & 6 & 1 & 1 & 5 & $<R L$ & 5 \\
\hline Silica (mg/L) & 47 & 68 & 94 & 39 & 26 & 56 & 39 & 39 & 43 & 53 & 51 \\
\hline Sodium (mg/L) & 67 & 930 & 1400 & 92 & 120 & 1400 & 160 & 140 & 210 & 490 & 820 \\
\hline Sulfate $(\mathrm{mg} / \mathrm{L})$ & 69 & 820 & 170 & 110 & 140 & 120 & 160 & 240 & 170 & 160 & 65 \\
\hline Thallium ( $\mu \mathrm{g} / \mathrm{L})$ & $<\mathrm{RL}$ & $<\mathrm{RL}$ & $<\mathrm{RL}$ & $<\mathrm{RL}$ & $<\mathrm{RL}$ & $<\mathrm{RL}$ & $<\mathrm{RL}$ & $<\mathrm{RL}$ & $<\mathrm{RL}$ & $<\mathrm{RL}$ & $<\mathrm{RL}$ \\
\hline Tungsten $(\mu \mathrm{g} / \mathrm{L})$ & 3 & 4 & 53 & 1 & 2 & 34 & 1 & 1 & 6 & $<R L$ & 100 \\
\hline Zinc (mg/L) & $<\mathrm{RL}$ & $<\mathrm{RL}$ & 0.17 & $<\mathrm{RL}$ & $<R L$ & $<\mathrm{RL}$ & $<\mathrm{RL}$ & $<\mathrm{RL}$ & $<\mathrm{RL}$ & $<\mathrm{RL}$ & $<\mathrm{RL}$ \\
\hline$\delta^{18} \mathrm{O}$ (VSMOW \%o) & -10.2 & -11.33 & -12.43 & -9.58 & -9.41 & -12.54 & -10.93 & -10.63 & -10.64 & -10.43 & -14.95 \\
\hline$\delta^{2} \mathrm{H}(\mathrm{VSMOW} \%$ ) & -87.6 & -95.8 & -109.2 & -84.8 & -84.2 & -110.6 & -92.3 & -89 & -88.8 & -89.5 & -116.8 \\
\hline
\end{tabular}


Table 3. Chemical analyses of well water samples (continued).

\begin{tabular}{|c|c|c|c|c|c|c|c|c|c|c|c|}
\hline Sample & 26 & 27 & 28 & 29 & 30 & 32 & 33 & 34 & 35 & 36 & 37 \\
\hline Field Temp $\left({ }^{\circ} \mathrm{C}\right)$ & 18.72 & 19.52 & 16.19 & 16.7 & 22.48 & 16.23 & 17.51 & 16.51 & 17.25 & 19.83 & 14.76 \\
\hline $\mathrm{pH}(\mathrm{SU})$ & 8.18 & 8.03 & 8.04 & 8.15 & 8.03 & 8.2 & 8.22 & 8.14 & 8.13 & 7.96 & 8.1 \\
\hline $\mathrm{pH}$ Temperature $\left({ }^{\circ} \mathrm{C}\right)$ & 20.6 & 21.2 & 20.7 & 20.8 & 21.1 & 21.1 & 20.9 & 20.7 & 20.7 & 20.7 & 20.7 \\
\hline Electrical Conductivity ( $\mu \mathrm{S} / \mathrm{cm})$ & 930 & 1300 & 1800 & 1900 & 1100 & 980 & 980 & 1600 & 1300 & 780 & 1700 \\
\hline Total Dissolved Solids (mg/L) & 601 & 860 & 1174 & 1286 & 742 & 614 & 634 & 1034 & 824 & 480 & 1132 \\
\hline Hardness (mg/L) & 311 & 281 & 390 & 293 & 340 & 374 & 307 & 469 & 299 & 192 & 402 \\
\hline Turbidity (NTU) & 0.2 & 0.2 & 0.2 & 0.55 & 0.6 & 0.2 & 0.2 & 0.2 & 1 & 0.2 & 0.2 \\
\hline Color (CU) & 5 & 20 & 15 & 20 & 15 & 1.5 & 7 & 5 & 7 & 5 & 15 \\
\hline Alkalinity as $\mathrm{CaCO}_{3}(\mathrm{mg} / \mathrm{L})$ & 278 & 330 & 378 & 422 & 266 & 177 & 287 & 264 & 308 & 251 & 490 \\
\hline Antimony $(\mu \mathrm{g} / \mathrm{L})$ & $<\mathrm{RL}$ & $<\mathrm{RL}$ & $<\mathrm{RL}$ & $<\mathrm{RL}$ & $<\mathrm{RL}$ & $<\mathrm{RL}$ & $<\mathrm{RL}$ & $<\mathrm{RL}$ & $<\mathrm{RL}$ & $<\mathrm{RL}$ & $<\mathrm{RL}$ \\
\hline Arsenic $(\mu \mathrm{g} / \mathrm{L})$ & 16 & 19 & 9 & 9 & 15 & 12 & 71 & 26 & 54 & 16 & 16 \\
\hline Arsenic III $(\mu \mathrm{g} / \mathrm{L})$ & $<R L$ & $<R L$ & $<R L$ & $<R L$ & $<R L$ & $<R L$ & $<R L$ & $<R L$ & $<R L$ & $<R L$ & $<R L$ \\
\hline Barium (mg/L) & 0.19 & 0.14 & 0.1 & 0.03 & 0.16 & 0.09 & 0.04 & 0.21 & 0.1 & 0.1 & 0.11 \\
\hline Bicarbonate (mg/L) & 339 & 403 & 461 & 514 & 324 & 216 & 350 & 322 & 376 & 306 & 598 \\
\hline Boron (mg/L) & 0.6 & 1.1 & 1.4 & 1.8 & 0.9 & 0.4 & 0.6 & 0.9 & 1.1 & 0.7 & 1.5 \\
\hline Calcium (mg/L) & 95 & 78 & 110 & 81 & 100 & 110 & 80 & 140 & 90 & 54 & 110 \\
\hline Carbonate $(\mathrm{mg} / \mathrm{L})$ & $<\mathrm{RL}$ & $<\mathrm{RL}$ & $<\mathrm{RL}$ & $<\mathrm{RL}$ & $<\mathrm{RL}$ & $<\mathrm{RL}$ & $<\mathrm{RL}$ & $<\mathrm{RL}$ & $<\mathrm{RL}$ & $<\mathrm{RL}$ & $<\mathrm{RL}$ \\
\hline Cesium $(\mu \mathrm{g} / \mathrm{L})$ & $<R L$ & $<\mathrm{RL}$ & $<\mathrm{RL}$ & $<\mathrm{RL}$ & $<\mathrm{RL}$ & $<\mathrm{RL}$ & $<\mathrm{RL}$ & $<\mathrm{RL}$ & $<\mathrm{RL}$ & $<\mathrm{RL}$ & $<\mathrm{RL}$ \\
\hline Chloride (mg/L) & 56 & 88 & 180 & 140 & 76 & 110 & 43 & 250 & 98 & 42 & 83 \\
\hline Copper (mg/L) & $<\mathrm{RL}$ & $<\mathrm{RL}$ & $<\mathrm{RL}$ & $<\mathrm{RL}$ & 0.03 & $<\mathrm{RL}$ & $<\mathrm{RL}$ & $<\mathrm{RL}$ & $<\mathrm{RL}$ & $<\mathrm{RL}$ & 0.02 \\
\hline Fluoride (mg/L) & 0.2 & 0.2 & $<\mathrm{RL}$ & 0.2 & 0.4 & 0.2 & 0.4 & $<R L$ & 0.6 & 0.4 & 0.4 \\
\hline Hydroxide (mg/L) & $<R L$ & $<R L$ & $<\mathrm{RL}$ & $<R L$ & $<R L$ & $<R L$ & $<\mathrm{RL}$ & $<R L$ & $<R L$ & $<R L$ & $<R L$ \\
\hline Iron (mg/L) & $<\mathrm{RL}$ & $<\mathrm{RL}$ & $<\mathrm{RL}$ & $<\mathrm{RL}$ & $<\mathrm{RL}$ & $<\mathrm{RL}$ & $<\mathrm{RL}$ & $<\mathrm{RL}$ & 0.23 & $<\mathrm{RL}$ & $<\mathrm{RL}$ \\
\hline Lithium $(\mu \mathrm{g} / \mathrm{L})$ & 0.09 & 0.1 & 0.11 & 0.12 & 0.1 & 0.07 & 0.14 & 0.1 & 0.11 & 0.1 & 0.13 \\
\hline Magnesium (mg/L) & 18 & 21 & 28 & 22 & 22 & 24 & 26 & 29 & 18 & 14 & 31 \\
\hline Manganese (mg/L) & 0.02 & 0.41 & 1.6 & 0.88 & 0.02 & $<\mathrm{RL}$ & 0.03 & 2.2 & 0.7 & 0.19 & 2.5 \\
\hline Mercury $(\mu \mathrm{g} / \mathrm{L})$ & $<R L$ & $<R L$ & $<\mathrm{RL}$ & $<\mathrm{RL}$ & $<\mathrm{RL}$ & $<\mathrm{RL}$ & $<\mathrm{RL}$ & $<\mathrm{RL}$ & $<\mathrm{RL}$ & $<\mathrm{RL}$ & $<R L$ \\
\hline Nitrate + Nitrite as N (mg/L) & 2.4 & 2.1 & 5 & 2.4 & 5.2 & 1.8 & 9.5 & $<\mathrm{RL}$ & 0.7 & 0.8 & 2.2 \\
\hline Potassium (mg/L) & 7 & 12 & 10 & 12 & 6 & 7 & 16 & 8 & 6 & $<R L$ & 6 \\
\hline Selenium $(\mu \mathrm{g} / \mathrm{L})$ & $<\mathrm{RL}$ & 2 & $<\mathrm{RL}$ & 2 & $<R L$ & 4 & $<\mathrm{RL}$ & $<R L$ & 1 & $<R L$ & $<R L$ \\
\hline Silica (mg/L) & 32 & 41 & 30 & 43 & 34 & 30 & 36 & 47 & 34 & 43 & 41 \\
\hline Sodium (mg/L) & 85 & 170 & 250 & 330 & 120 & 66 & 95 & 160 & 160 & 100 & 230 \\
\hline Sulfate (mg/L) & 120 & 220 & 270 & 390 & 190 & 140 & 130 & 210 & 210 & 90 & 310 \\
\hline Thallium ( $\mu \mathrm{g} / \mathrm{L})$ & $<\mathrm{RL}$ & $<\mathrm{RL}$ & $<\mathrm{RL}$ & $<\mathrm{RL}$ & $<\mathrm{RL}$ & $<\mathrm{RL}$ & $<\mathrm{RL}$ & $<\mathrm{RL}$ & $<\mathrm{RL}$ & $<\mathrm{RL}$ & $<\mathrm{RL}$ \\
\hline Tungsten $(\mu \mathrm{g} / \mathrm{L})$ & $<R L$ & $<R L$ & $<R L$ & $<R L$ & $<R L$ & $<R L$ & $<R L$ & 2 & 2 & $<R L$ & 2 \\
\hline Zinc (mg/L) & 0.05 & $<\mathrm{RL}$ & $<\mathrm{RL}$ & $<\mathrm{RL}$ & 0.05 & $<\mathrm{RL}$ & $<\mathrm{RL}$ & $<\mathrm{RL}$ & $<\mathrm{RL}$ & 0.05 & $<\mathrm{RL}$ \\
\hline$\delta^{18} \mathrm{O}$ (VSMOW \%о) & -10.59 & -10.93 & -10.7 & -10.69 & -10.77 & -11.48 & -10.88 & -10.85 & -10.96 & -10.25 & -10.89 \\
\hline$\delta^{2} \mathrm{H}(\mathrm{VSMOW} \% \mathrm{)}$ & -89.2 & -90.3 & -89.7 & -89.9 & -90.4 & -93.7 & -90.5 & -90.5 & -91 & -87 & -91.3 \\
\hline
\end{tabular}


Table 3. Chemical analyses of well water samples (continued).

\begin{tabular}{|c|c|c|c|c|c|c|c|}
\hline Sample & 38 & 39 & 40 & 41 & 42 & P1 & P2 \\
\hline Field Temp $\left({ }^{\circ} \mathrm{C}\right)$ & 85 & 82 & 21.98 & 79 & 14.22 & 139.44 & 142.33 \\
\hline $\mathrm{pH}(\mathrm{SU})$ & 7.91 & 7.85 & 8.19 & 7.9 & 8.14 & 8.25 & 7.51 \\
\hline $\mathrm{pH}$ Temperature $\left({ }^{\circ} \mathrm{C}\right)$ & 20.8 & 20.8 & 20.8 & 20.8 & 20.9 & 18.8 & 19.4 \\
\hline Electrical Conductivity ( $\mu \mathrm{S} / \mathrm{cm})$ & 7100 & 7200 & 2300 & 7100 & 1800 & 8000 & 7500 \\
\hline Total Dissolved Solids (mg/L) & 4040 & 4092 & 1542 & 4120 & 1160 & 4815 & 4424 \\
\hline Hardness (mg/L) & 205 & 182 & 472 & 182 & 318 & 177 & 177 \\
\hline Turbidity (NTU) & 3.8 & 0.2 & 0.2 & 0.2 & 0.45 & 0.7 & 0.9 \\
\hline Color (CU) & 12 & 7 & 7 & 5 & 5 & 5 & 5 \\
\hline Alkalinity as $\mathrm{CaCO}_{3}(\mathrm{mg} / \mathrm{L})$ & 112 & 131 & 375 & 103 & 520 & 58 & 70 \\
\hline Antimony ( $\mu \mathrm{g} / \mathrm{L})$ & 2 & 2 & 2 & $<R L$ & $<R L$ & $<\mathrm{RL}$ & 2 \\
\hline Arsenic $(\mu \mathrm{g} / \mathrm{L})$ & 4 & 7 & 160 & $<R L$ & 52 & 18 & 26 \\
\hline Arsenic III ( $\mu \mathrm{g} / \mathrm{L})$ & $<R L$ & 9 & $<R L$ & $<R L$ & $<R L$ & 34 & 20 \\
\hline Barium (mg/L) & 0.2 & 0.24 & 0.09 & 0.18 & 0.05 & 0.08 & 0.14 \\
\hline Bicarbonate (mg/L) & 136 & 159 & 457 & 126 & 634 & 71 & 85 \\
\hline Boron (mg/L) & 15 & 15 & 2 & 16 & 1.8 & 23 & 18 \\
\hline Calcium (mg/L) & 82 & 73 & 110 & 73 & 78 & 71 & 71 \\
\hline Carbonate (mg/L) & $<R L$ & $<R L$ & $<R L$ & $<R L$ & $<R L$ & $<R L$ & $<\mathrm{RL}$ \\
\hline Cesium ( $\mu \mathrm{g} / \mathrm{L})$ & 170 & 130 & $<R L$ & 170 & $<R L$ & 53 & 130 \\
\hline Chloride (mg/L) & 2200 & 2200 & 370 & 2200 & 110 & 2600 & 2400 \\
\hline Copper (mg/L) & 0.02 & $<\mathrm{RL}$ & $<R L$ & $<R L$ & $<\mathrm{RL}$ & $<R L$ & $<R L$ \\
\hline Fluoride (mg/L) & 4.1 & 4.4 & 0.8 & 4.3 & 1.2 & 3.2 & 4.8 \\
\hline Hydroxide (mg/L) & $<R L$ & $<R L$ & $<R L$ & $<R L$ & $<R L$ & $<R L$ & $<\mathrm{RL}$ \\
\hline Iron (mg/L) & 0.66 & 0.11 & $<R L$ & 0.1 & 0.15 & 0.55 & 0.59 \\
\hline Lithium ( $\mu \mathrm{g} / \mathrm{L})$ & 2 & 2 & 0.38 & 2 & 0.24 & 3.8 & 2.2 \\
\hline Magnesium (mg/L) & $<\mathrm{RL}$ & $<R L$ & 48 & $<R L$ & 30 & $<\mathrm{RL}$ & $<\mathrm{RL}$ \\
\hline Manganese (mg/L) & 0.12 & 0.25 & 0.04 & 0.04 & 0.62 & 0.02 & 0.03 \\
\hline Mercury ( $\mu \mathrm{g} / \mathrm{L})$ & $<R L$ & $<R L$ & $<\mathrm{RL}$ & $<R L$ & $<R L$ & $<R L$ & $<\mathrm{RL}$ \\
\hline Nitrate + Nitrite as N (mg/L) & $<R L$ & $<R L$ & 3.9 & $<R L$ & $<R L$ & $<R L$ & $<R L$ \\
\hline Potassium (mg/L) & 28 & 45 & 21 & 30 & 9 & 68 & 100 \\
\hline Selenium ( $\mu \mathrm{g} / \mathrm{L})$ & 4 & 4 & 4 & 6 & $<R L$ & 5 & 11 \\
\hline Silica (mg/L) & 111 & 120 & 62 & 103 & 45 & 158 & 145 \\
\hline Sodium (mg/L) & 1300 & 1400 & 340 & 1500 & 290 & 1600 & 1500 \\
\hline Sulfate (mg/L) & 160 & 150 & 280 & 170 & 270 & 180 & 180 \\
\hline Thallium ( $\mu \mathrm{g} / \mathrm{L})$ & $<R L$ & $<R L$ & $<R L$ & $<R L$ & $<R L$ & 4 & 0.9 \\
\hline Tungsten ( $\mu \mathrm{g} / \mathrm{L})$ & 56 & 56 & 3 & 55 & $<\mathrm{RL}$ & 47 & 48 \\
\hline Zinc (mg/L) & 0.06 & $<R L$ & $<R L$ & $<R L$ & $<R L$ & $<R L$ & $<R L$ \\
\hline$\delta^{18} \mathrm{O}(\mathrm{VSMOW} \%$ \%) & -12.5 & -12.56 & -10.45 & -12.62 & -10.41 & -11.06 & -11.95 \\
\hline$\delta^{2} \mathrm{H}(\mathrm{VSMOW} \%$ \%) & -110.7 & -110.9 & -89.6 & -110.7 & -88.7 & -104.4 & -108.9 \\
\hline
\end{tabular}


Table 4. Quality assurance data. "dup" = duplicate, "std dev" = standard deviation between (btwn) the sample and its duplicate.

\begin{tabular}{|c|c|c|c|c|c|c|c|c|c|}
\hline Sample \# & 20 & 18 & $\%$ relative & 24 & $\%$ relative & 31 & \%relative & 43 & $\%$ relative \\
\hline description & field blank & dup of \#17 & $\begin{array}{c}\text { std dev } \\
\text { btwn } 17 \& 18\end{array}$ & dup of \#23 & $\begin{array}{c}\text { std dev } \\
\text { btwn } 23 \& 24\end{array}$ & dup of \#30 & $\begin{array}{c}\text { std dev } \\
\text { btwn } 30 \& 31\end{array}$ & dup of \#42 & $\begin{array}{c}\text { std dev } \\
\text { btwn } 42 \& 43\end{array}$ \\
\hline Alkalinity as $\mathrm{CaCO} 3$ (mg/L) & 2 & 156 & 1 & 527 & 0 & 269 & 1 & 523 & 0 \\
\hline Antimony ( $\mu \mathrm{g} / \mathrm{L})$ & $<\mathrm{RL}$ & $<\mathrm{RL}$ & & $<\mathrm{RL}$ & & $<\mathrm{RL}$ & & $<\mathrm{RL}$ & $<\mathrm{RL}$ \\
\hline Arsenic $(\mu \mathrm{g} / \mathrm{L})$ & $<\mathrm{RL}$ & 9 & 0 & 170 & 0 & 15 & 0 & 52 & 0 \\
\hline Arsenic III ( $\mu \mathrm{g} / \mathrm{L})$ & $<\mathrm{RL}$ & 10 & 7 & $<\mathrm{RL}$ & $<\mathrm{RL}$ & $<\mathrm{RL}$ & $<\mathrm{RL}$ & $<\mathrm{RL}$ & $<\mathrm{RL}$ \\
\hline Barium (mg/L) & $<\mathrm{RL}$ & 0.16 & 5 & 0.13 & 0 & 0.15 & 5 & 0.05 & 0 \\
\hline Bicarbonate (mg/L) & $<\mathrm{RL}$ & 190 & 2 & 643 & 0 & 328 & 1 & 638 & 0 \\
\hline Boron (mg/L) & $<\mathrm{RL}$ & 14 & 0 & 1.5 & 5 & 0.9 & 0 & 1.9 & 4 \\
\hline Calcium (mg/L) & $<\mathrm{RL}$ & 59 & 1 & 160 & 0 & 98 & 1 & 80 & 2 \\
\hline Carbonate (mg/L) & $<R L$ & $<R L$ & $<\mathrm{RL}$ & $<\mathrm{RL}$ & $<R L$ & $<R L$ & $<R L$ & $<\mathrm{RL}$ & $<\mathrm{RL}$ \\
\hline Cesium ( $\mu \mathrm{g} / \mathrm{L})$ & $<\mathrm{RL}$ & $<\mathrm{RL}$ & $<\mathrm{RL}$ & $<\mathrm{RL}$ & $<\mathrm{RL}$ & $<\mathrm{RL}$ & $<\mathrm{RL}$ & $<\mathrm{RL}$ & $<\mathrm{RL}$ \\
\hline Chloride (mg/L) & $<\mathrm{RL}$ & 2200 & 3 & 800 & 3 & 78 & 2 & 110 & 0 \\
\hline Copper (mg/L) & $<\mathrm{RL}$ & $<\mathrm{RL}$ & $<\mathrm{RL}$ & $<\mathrm{RL}$ & $<\mathrm{RL}$ & 0.03 & 0 & $<\mathrm{RL}$ & $<\mathrm{RL}$ \\
\hline Fluoride (mg/L) & $<\mathrm{RL}$ & 2.4 & 0 & 0.8 & 0 & 0.4 & 0 & 1.2 & 0 \\
\hline Hydroxide (mg/L) & $<R L$ & $<R L$ & $<R L$ & $<\mathrm{RL}$ & $<R L$ & $<R L$ & $<R L$ & $<\mathrm{RL}$ & $<\mathrm{RL}$ \\
\hline Iron (mg/L) & $<R L$ & 0.09 & 35 & 0.05 & 0 & 0.11 & $<R L$ & 0.15 & 0 \\
\hline Lithium ( $\mu \mathrm{g} / \mathrm{L})$ & $<R L$ & 1.5 & 5 & 0.21 & 0 & 0.1 & 0 & 0.23 & 3 \\
\hline Magnesium (mg/L) & $<R L$ & 7 & 0 & 62 & 1 & 22 & 0 & 31 & 2 \\
\hline Manganese (mg/L) & $<\mathrm{RL}$ & 0.26 & 0 & $<\mathrm{RL}$ & $<R L$ & 0.02 & 0 & 0.63 & 1 \\
\hline Mercury $(\mu \mathrm{g} / \mathrm{L})$ & $<\mathrm{RL}$ & $<R L$ & $<R L$ & $<\mathrm{RL}$ & $<R L$ & $<R L$ & $<R L$ & $<\mathrm{RL}$ & $<R L$ \\
\hline Nitrate+Nitrite as N (mg/L) & $<\mathrm{RL}$ & $<R L$ & $<\mathrm{RL}$ & 0.5 & 13 & 5.1 & 1 & $<\mathrm{RL}$ & $<R L$ \\
\hline Potassium (mg/L) & $<\mathrm{RL}$ & 24 & 6 & 12 & 0 & $<R L$ & $<R L$ & 9 & 0 \\
\hline Selenium ( $\mu \mathrm{g} / \mathrm{L})$ & $<R L$ & 4 & 28 & $<\mathrm{RL}$ & $<R L$ & $<R L$ & $<R L$ & $<\mathrm{RL}$ & $<\mathrm{RL}$ \\
\hline Silica (mg/L) & $<R L$ & 58 & 2 & 53 & 0 & 34 & 0 & 47 & 3 \\
\hline Sodium (mg/L) & $<\mathrm{RL}$ & 1400 & 0 & 490 & 0 & 110 & 6 & 300 & 2 \\
\hline Sulfate (mg/L) & $<R L$ & 110 & 6 & 160 & 0 & 190 & 0 & 260 & 3 \\
\hline Thallium ( $\mu \mathrm{g} / \mathrm{L})$ & $<\mathrm{RL}$ & $<R L$ & $<R L$ & $<\mathrm{RL}$ & $<R L$ & $<R L$ & $<R L$ & $<\mathrm{RL}$ & $<\mathrm{RL}$ \\
\hline Tungsten ( $\mu \mathrm{g} / \mathrm{L})$ & $<R L$ & 34 & 0 & $<\mathrm{RL}$ & $<\mathrm{RL}$ & $<R L$ & $<R L$ & $<\mathrm{RL}$ & $<R L$ \\
\hline Zinc (mg/L) & $<\mathrm{RL}$ & $<R L$ & $<\mathrm{RL}$ & $<\mathrm{RL}$ & $<R L$ & 0.05 & 0 & $<\mathrm{RL}$ & $<\mathrm{RL}$ \\
\hline$\delta^{18} \mathrm{O}$ (VSMOW \%o) & & -12.5 & 0 & -10.46 & 0 & -10.76 & 0 & -10.38 & 0 \\
\hline$\delta^{2} \mathrm{H}(\mathrm{VSMOW} \%$ ) & & -110.8 & 0 & -89.2 & 0 & -89.8 & 0 & -88 & -1 \\
\hline
\end{tabular}




\section{Acknowledgments}

Dr. James Carr, University of Nevada, contributed to the sampling design. The Stable Isotope Laboratory at the University of Nevada, managed by Dr. Simon Poulson, provided prompt and thorough analyses of samples. Residents in the Stillwater area of Churchill County, NV were kind enough to allow us access to wells. Personnel at the Ormat geothermal power plants in Churchill County provided access to geothermal sources and assistance with obtaining samples. Finally, we thank Mike Lico and Ralph Seiler of the U.S. Geological Survey for their detailed and prompt reviews. This project is based upon work supported by the Cooperative State Research, Education, and Extension Service, U.S. Department of Agriculture, under Agreement No. [200251130-01944]. Stillings' time was supported by the U.S. Geological Survey, Mineral Resources Program.

\section{References Cited}

American Public Health Association, American Water Works Association, Water Pollution Control Federation, \& Water Environment Federation, 1998, Standard Methods for the Examination of Water and Wastewater: including bottom sediments and sludges $\left(20^{\text {th }} \mathrm{ed}\right.$.): Washington, D.C., American Public Health Association.

Epstein, S., and Mayeda, T., 1953, Variation of $\mathrm{O}^{18}$ content of waters from natural sources: Geochimica et Cosmochimica Acta, v. 4, p. 213-224.

Fosbury, D, 2007, Identifying the influences of geothermal sources on shallow aquifer water quality: Reno, NV, University of Nevada, Reno, M.S. thesis, 66 p.

Garside, L.J., and Schilling, J.H., 1979, Thermal waters of Nevada: Nevada Bureau of Mines and Geology Bulletin 91, 168 p.

Glancy, P.A., 1986, Geohydrology of the basalt and unconsolidated sedimentary aquifers in the Fallon area, Churchill County, Nevada: U.S. Geological Survey Water Supply Paper 2263, $62 \mathrm{p}$.

Lico, M.S., Welch, A.H., and Hughes, J.L., 1987, Geochemistry of ground water in the shallow alluvial aquifer, Carson Desert, western Nevada, in: Averett, R.C., and McKnight, D.M., (eds.), Chemical Quality of Water and the Hydrologic Cycle: Chelsea, MI, Lewis Publishers, p. 89-109.

Lico, M.S., and Seiler, R.L., 1994, Ground-water quality and geochemistry, Carson Desert, western Nevada, U.S. Geological Survey Open File Report 94-0031, 91 p.

Morrison, J., Brockwell, T., Merren, T., Fourel, F., and Phillips, A.M., 2001, On-line highprecision stable hydrogen isotopic analyses on nanoliter water samples: Analytical Chemistry, v. 73, p. 3570-3575.

Seiler, R.L., Stollenwerk, K.G., and Garbarino, J.R., 2005, Factors controlling tungsten concentrations in ground water, Carson Desert, Nevada:. Applied Geochemistry, v. 20, p. 423-441.

Skoog D.A., West D.M., Holler, F.A., and Crouch, S. R., 2000, Analytical chemistry: An introduction ( $7^{\text {th }}$ ed.): Fort Worth, TX, Saunders College Pub., 773 p.

U.S. Environmental Protection Agency, website accessed May, 2008, Clean Water Act Analytical Test Methods: http://www.epa.gov/waterscience/methods/ 
Welch, A.H., and Lico, M.S., 1998, Factors controlling As and U in shallow ground water, southern Carson Desert, Nevada: Applied Geochemistry, v. 13, p. 521-539.

Webster, J.G., and Nordstrom, D.K., 2003, Geothermal arsenic; the source, transport and fate of arsenic in geothermal systems, in: Stollenwerk, K.G., (ed.), Arsenic in Ground Water: Boston, MA, Kluwer Academic Publishers, p. 101-125. 